\title{
DEPRESIÓN EN LA VEJEZ: EVALUACIÓN, VARIABLES IMPLICADAS Y RELACIÓN CON EL DETERIORO COGNITIVO
}

\author{
José Miguel Latorre Postigo \\ Juan Montanés Rodriguez \\ Universidad de Castilla-La Mancha
}

\begin{abstract}
RESUMEN
Se ha evaluado la depresión y el deterioro cognitivo en una muestra de ancianos con una un conjunto de pruebas que incluye tests neuropsicológicos, la escala de depresión del Centro de Estudios Epidemiologicos (CES-D) y la que utiliza los críterios del DSM-III-R. Las dos escalas muestran un gran coincidencia en la evaluación de la depresión. La prevalencia de la depresión disminuye con la edad hasta los 80 años, momento en el que se invierte la tendencia, es mayor en las mujeres, en los que viven en una residencia y en los que tienen un nivel de estudios más bajo. A partir de los 80 anos aumenta el diagnóstico conjunto depresión-demencia y la probabilldad de confusión en el mismo, sobre todo en los sujetos con un nivel de estudios bajo, por lo que se apunta la necesidad de que en los estudios sobre la depresión en la vejez se incluyan pruebas neuropsicológicas, especialmente de recuerdo demorado, fluidez verbal, memoria lógica y metamemoria, para diferenciar ambos estados.
\end{abstract}

Palabras clave: Depresión, demencia, vejez, CES-D, DSM-III-R, evaluación neuropsicológica.

\section{ABSTRACT}

It has been evaluated the depression and the cognitive impaiment in an sample of elderly subjects with a set of tests that include neuropsychological tests, the Center of Epidemiological Studies Depression scale (CES-D) and the one which uses the criteria of DSM-III-R. The two scales show a great coincidence in the evaluation of depression. The prevalence of depression decreasses with the age until 80 years, moment in wich the trend is invested, it is greater in woman, in those who live in a residence and those who have a lower studies level. After 80 years increases the diganostic joint depressioncognitive and the probability of confusion in the same, above all in the subjects with a studies level less. Therefore, it is noted the need of include neuropsychological tests in the studies on depression in aging, fundamentally delayed recall, verbal fluency, logical memory and metamemory tests, to differentiate both states.

Key words: depression, aging, cognitive impairments, CES-D, DSM-III-R, neuropsychological assessment. 


\section{INTRODUCCIÓN}

La naturaleza de la depresión en la vejez parece ser fenomenológicamente distinta a la de otras edades. A ello contribuye sin duda la especial asociación de la depresión con el deterioro cognitivo en este periodo de la vida, que puede producir una importante confusión en la labor clínica con los ancianos. Una interesante línea de investigación en esta área, por con-siguiente, es la que combina la evaluación clínica de la depresión y la demencia con datos procedentes de pruebas neuropsicológicas con el fin de discriminar mediante éstas depresión, demencia y envejecimiento normal.

Nuestro proposito es evaluar la depresión mediante dos escalas, una clinica y otra por autoinforme, para asi detectar el grado de coincidencia en la evaluación de la depresión de ambas escalas, y relacionar los resultados con el deterioro cognitivo en la vejez y con algunas variables implicadas en esa relación como son la edad, el género, el nivel de estudios y el lugar de residencia.

Actualmente no existe unanimidad sobre el concepto de depresión. En muchas ocasiones, los investigadores no comparten el mismo significado para este concepto y de hecho lo utilizan para referirse a situaciones muy heterogéneas (Rodríguez-Naranjo y Godoy, 1997). La falta de unanimidad, sin duda, dificulta la comparación de investigaciones y resultados (Buendía y Riquelme, 1995), además de influir negativamente en el diagnóstico y tratamiento de la depresión en diferentes edades, especialmente en la vejez.

La diversidad de escalas que se usan en la evaluación de la depresión no ayuda precisamente a solucionar estas dificultades. Convencionalmente la forma más operativa de conceptualizar los trastornos mentales en la vejez, entre ellos la depresión, es la proporcionada por los resultados obtenidos por los sujetos en una escala clinica según criterios del DSM-III-R (American Psychiatric Association, 1987), actualmente DSM-IV (1994), así como por diversas escalas de autoinforme (Lebowitz y Niederehe, 1992), como la escala CES-D (Escala de Depresión del Centro de Estudios Epidemiológicos, Radloff, 1977). El Criterio de evaluación de los desórdenes depresivos, proporcionado por el DSM-III-R, es uno de los criterios más aceptados de evaluación de estos desórdenes. Y la CES-D es una de las escalas más utilizadas en contextos clínicos y de investigación, y tiene entre sus ventajas la de estar menos amenazada en su validez por la enfermedad física y las discapacidades (Berkman, Berkman, Kasl, Freeman y Leo, 1986; Gatz, Johansson, Pedersen, Berg y Reynolds, 1993), frente a otras medidas de autoinforme donde las complicaciones físicas están directamente reflejadas en elevadas puntuaciones en los items somáticos (Berry, Storandt, y Coyne, 1984). La escala CES-D se ha utilizado para la evaluación de la depresión en investigaciones comunitarias, incluyendo poblaciones de ancianos y de enfermos físicos (Roberts y Vernon, 1983; Krause, 1986; Radloff y Teri, 1986; Gatz y Hurwicz, 1990). Las áreas más frecuentes, entre otras, en las que se utiliza son: el seguimiento de sintomas depresivos en enfermos físicos (cáncer, enfermedades cardiovasculares, SIDA, etc.), la evaluación de la depresión durante el embarazo y el posparto, el estudio del impacto de la enfermedad de Alzheimer sobre los cuidadores, la detección de sintomas de depresión en las madres de niños enfermos y el diagnóstico de la depresión en ancianos. La utilización de la escala CES-D nos permite además comparar nuestros datos con los obte-nidos en diversos paises, con muestras de gran tamano de diferentes edades (García y Marks, 1989; Gatz y Hurwicz, 1990; Krause y Liang, 1992; Gatz et al., 1993).

Con los datos disponibles no se puede confirmar la creencia común de que la depresión aumenta con la edad y de que es mayor, en consecuencia, durante la vejez. Las investigaciones actuales sugieren dos posibilidades: Primera, que la depresión alcanza valores altos en la juventud, disminuye durante la adultez y vuelve a incrementarse en la vejez a valores similares a los de la juventud, de forma que la curva de la prevalencia a lo largo del ciclo vital tiene forma de U (Newmann, 1989; Gatz y Hurwicz, 1990). El incremento de la prevalencia durante la vejez se hace significativo a partir de los 75 años, en comparación con el intervalo de 55 a 74 años de edad (Murrell, Himmelfarb y Wright, 1983). Y segunda, que el riesgo más elevado de padecer un episodio depresivo se observa entre los jóvenes adultos y disminuye a partir de esta edad, dándose una curva descendente a partir de los 45 años 
(Vázquez y Sanz, 1995). Estas investigaciones, por tanto, no ofrecen suficiente evidencia empirica de que la prevalencia de la depresión sea mayor en la vejez o de que esta edad se caracterice, en comparación con otras edades, por una clara sintomatologia depresiva. Sin embargo, es habitual que la depresión se considere una de las alteraciones psicológicas de la misma (Buendia y Riquelme, 1995) o, incluso, el desorden funcional más común en la vejez (Kalish, 1991). Se ha sugerido al respecto que durante la vejez pueden aparecer altos niveles de síntomas depresivos sin que necesariamente alcancen el criterio para el diagnóstico de depresión mayor (Gatz, 1994), siendo la distimia, en consecuencia, más frecuente en los grupos de mayor edad. En este sentido, algunos trabajos, que han utilizado escalas de autoinforme de síntomas depresivos, apuntan a que un tercio de los sujetos tenlan disforia significativa sin alcanzar el criterio de un desorden depresivo mayor (Blazer, Hughes y George, 1987). No obstante, aunque no haya mayor tasa de depresión en la vejez, si que parece que la depresión en la última etapa de la vida es diferente a la de épocas más tempranas (Ruiz, Martin, Serrano y Morales, 1988; Gatz, 1994), de forma que durante la vejez la depresión normalmente se asocia a múltiples pérdidas orgánicas y cognitivas, y a diversas circunstancias.

Entre las caracteristicas diferenciales de la depresión en la vejez, en relación a otras edades, una muy frecuente es la queja de los ancianos deprimidos acerca de problemas de memoria, que enmascara su trastorno principal, acudiendo a las consultas médicas con la sospecha de problemas de demencia y no de depresión. Además, los ancianos con deterioro cognitivo presentan, en muchos casos, al mismo tiempo depresión (Reifier, Larson y Hanley, 1982). Esto nos indica la especial interacción entre las variables cognitivas y las afectivas durante la vejez, y la posibilidad de confusión en el diagnóstico.

La demencia se le define como un desorden de la memoria y la cognición, que empeora progresivamente (Gatz, 1994), aunque no está necesariamente asociada a la vejez (González y Ramos, 1995). Dado que en la vejez se incrementa progresivamente la demencia, la posibilidad de confusión en el diagnóstico diferencial entre ésta y la depre- sión se hace mayor. En este sentido pensamos que la comprensión de la naturaleza, severidad y causas exactas del deterioro cognitivo es importante para el diagnóstico y tratamiento de la depresión.

Los estudios sobre el funcionamiento cognitivo de los deprimidos han puesto de manifiesto consistentemente un deterioro en tareas atencionales y de memoria inmediata (Sweeney, Stokes, Wetzler y Kocsis, 1989). Los sujetos depresivos muestran un rendimiento inferior en las pruebas explicitas de memoria de recuerdo libre, recuerdo con claves y reconocimiento; sin embargo, en las pruebas implicitas de memoria las diferencias entre depresivos y no depresivos dependen de la naturaleza de las propias pruebas: si éstas requieren un nivel de procesamiento superficial no hay diferencias, mientras que si existen diferencias si se requiere un nivel de procesamiento profundo, semejante al requerido en las pruebas explicitas (Beato y Fernández, 1995). Estas diferencias en el rendimiento cognitivo entre depresivos y no depresivos son significativamente mayores en los ancianos (Deptula, Singh, Pomara, 1993).

La relación entre los estados de depresión y problemas de memoria $y$ atencionales ha hecho que estos problemas sean incluidos entre los criterios de evaluación clinica de la depresión. Sin embargo, no está clara esta relación. Se aventura como posible explicación el hecho de que la depresión pueda estar relacionada con problemas atencionales, que afectan a la fase inicial de la adquisición y codificación de la información. Lo cual se confirma en estudios psicofisiológicos en los que se muestra una reducción en el nivel de reactividad a la estimulación sensorial en los deprimidos (Dawson, Schell y Catania, 1977). Otras posibles explicaciones son el deterioro de los recursos cognitivos (Ellis y Ashbrook, 1988), las dificultades en la elaboración del material (Watkins, Matheuws, Williamson y Fuller, 1992) y las estrategias ineficaces de organización subjetiva (Davis, 1979).

Diversos trabajos apuntan que la depresión es el mejor predictor de la percepción de problemas de memoria (metamemoria) en la vejez (Zelinski et al., 1980; Bazargan y Barbre, 1994; McDougall, 1994; Dellefield y McDougall, 1996), por lo que, a su vez, la metamemoria se convierte en un indicador importante para diferenciar deprimidos y no deprimidos. La implicación de 
la metamemoria en el rendimiento mnésico de los ancianos es origen de cierta controversia. Para algunos autores las quejas de memoria de los ancianos no se corresponden con el rendimiento posterior de los mismos (O'Connor, Pollit y Roth 1990; Bermejo y Garcla de Blas, 1993). Otros, sin embargo, sugieren la existencia de una importante relación entre la metamemoria y la ejecución en tareas de memoria, especialmente en la vejez (Zelinski, Gilewski y Thompson, 1980; Dellefield y McDougall, 1996; Guirado y Latorre, 1996). Algunos autores, incluso, no sólo han encontrado relación entre la metacognición y la memoria, sino también entre aquélla y el rendimiento en diversas tareas cognitivas (Fingerman y Perimutter, 1994). Esta relación es superior conforme el sujeto es más viejo.

Además de la metamemoria, que diferencia entre sujetos deprimidos y normales, como ya hemos senalado, existen otras tareas que han mostrado su eficacia a la hora de distinguir entre depresión y demencia. Entre estas, destaca la capacidad de retención de la información (recuerdo demorado), la cual se ha comprobado que se mantiene más o menos intacta en los ancianos deprimidos y se deteriora considerablemente en la demencia tipo Alzheimer (Massan, Butters y Delis, 1994).

En cuanto al género, nuestro objetivo es ver si se confirma lo que otros trabajos ya apuntan acerca de que las mujeres presentan mayor tasa de depresión en todas las edades, siendo estas diferencias mayores en la vejez (Gatz, 1994; Gatz y Hurwicz, 1990). A este fenómeno se le han dado diversas explicaciones, que van desde lo biológico a lo sociocultural, y sigue siendo bastante controvertido (Huyck, 1990). Pero dejando a un lado los resultados contradictorios, lo cierto es que el balance de la evidencia clínica y epidemiológica indica que la mujer presenta un riesgo 1.5 veces mayor que el hombre de sufrir trastornos depresivos o depresión mayor (Weissman y Klerman, 1985; Bebbington, 1988; Anthony y Aboraya, 1992).

Por otra parte, si la edad es un factor de riesgo para la demencia, se puede afirmar que la educación es un factor protector: parece ser que a niveles educativos más bajos existen mayores riesgos de deterioro cognitivo en general (Steen, 1992; Bermejo y Colmenarejo, 1993; Mortimer, 1993; Gatz, 1994), y en concreto de la memoria en los ancianos (Colsher y Wallace, 1991). A esto se le han dado diversas explicaciones, desde la que afirma que el nivel de estudios propicia una mayor actividad cerebral, que protegeria en la vejez del deterioro cognitivo (Zhang, Katzman y Salmon, 1990), a la que asegura que la educación serla un indicador del nivel socioeconómico del sujeto, factor de riesgo verdaderamente implicado en el deterioro cognitivo (Bermejo y Colmenarejo, 1993). Esta relación entre nivel educativo y demencia, independientemente de cual sea su origen, nos hace presumir la existencia de una interrelación entre el nivel de estudios y la depresión, que pretendemos confirmar en nuestro trabajo. Al respecto, en algunos estudios ya se ha establecido que un bajo nivel de estudios es un factor de riesgo para la presencia de la depresión en la edad adulta y la vejez (Anthony y Aboralla, 1992).

En cuanto al lugar de residencia, hemos de señalar que durante la vejez muchos ancianos siguen viviendo en sus domicilios 0 en el de familiares, pero otros muchos se ven obligados a salir de su ambiente habitual y a vivir en otro extraño, con las posibles consecuencias negativas que ello puede implicar. Vivir en una residencia se puede convertir en un suceso estresante que se acumula a otros durante la vejez, y que influye de forma distinta en función del ambiente institucional que exista, ya que la diversidad de ambientes residenciales no permite una interpretación univoca de esta cuestión (Fernández-Ballesteros, Izal, Montorio, González y Dlaz, 1992). No obstante, diversos trabajos apuntan hacia una mayor prevalencia de la depresión en ancianos institucionalizados (Busse, 1992). En este sentido, distintos autores han encontrado que un gran número de ancianos de las residencias presentan algún trastorno mental diagnosticable, fundamentalmente demencia y depresión (Cohen, 1990; Liptzin, 1992). Sin embargo, pensamos que la presencia de problemas depende, en gran medida, de las caracteristicas de la "institución" residencial.

En resumen y de acuerdo con el marco conceptual desarrollado, a veces con datos contradictorios, nuestras predicciones en este trabajo se fundamentan en la especial interrelación entre la depresión, el deterioro cognitivo y otras variables como la edad, el género, el nivel de estudios y la residencia. Las predicciones especificas son: 
1) Las escalas por autoinforme, en este caso la CES-D, muestran un alto grado de coincidencia en la evaluación de la depresión en ancianos con la evaluación clínica según los criterios del DSM-III-R.

2) Durante la vejez a mayor edad mayor interrelación entre depresión y deterioro cognitivo, de forma que en los sujetos de más edad se incrementará la prevalencia conjunta de deterioro cognitivo y depresión.

3) La depresión es mayor en las mujeres que en los hombres durante la vejez en todos los intervalos de edad.

4) La depresión es mayor en los ancianos que viven en la residencia que en los que viven en su domicilio.

5) Las pruebas neuropsicológicas que mejor discriminan la depresión son: a) la metamemoria cuando se comparan los sujetos deprimidos con los normales; b) el recuerdo demorado cuando se comparan los sujetos con deterioro cognitivo con los deprimidos.

6) La capacidad discriminativa de las pruebas neuropsicologicas se modifica en función del nivel de estudios. Cuando los sujetos no poseen estudios la capacidad discriminativa de los tests disminuye, convirtiéndose en las más discriminativas las tareas de carácter verbal.

7) El nivel de estudios está implicado indirectamente en el desarrollo de la depresión a través del deterioro cognitivo, de forma que se da una mayor confusión entre deterioro cognitivo y depresión cuanto menor es el nivel de estudios.

Por último, conviene señalar que este trabajo no pretende ser un estudio epidemiológico sobre la prevalencia de la depresión en la vejez. Por tanto, los resultados en cuanto a las tasas de depresión en la vejez no son directamente extrapolables a la población en general.

\section{MÉTODO}

\section{Muestra}

La muestra está compuesta por un total de 230 sujetos de la ciudad de Albacete mayores de 60 años. De los sujetos entrevistados 101 $(44.3 \%)$ viven en una residencia de válidos del INSERSO y el resto en su domicilio particular y acuden a dos hogares del pensionista.

La residencia es un centro de 204 plazas, que sirve de vivienda permanente a personas mayores de 60 años, que pueden valerse por sl mismas en el momento del ingreso, y que por circunstancias económicas, familiares, etc., no pueden permanecer en su propio domicilio. Entre sus objetivos están: servir de vivienda permanente y común; prestar atención integral y continuada, de manera que se retarde en lo posible su incapacidad; y prestar cuidados cuando de produzca algún tipo de discapacidad.

Los sujetos fueron seleccionados con la ayuda de los trabajadores sociales de los tres centros, con criterios de representatividad, por edad y sexo, de esos centros. Todos los sujetos participaron voluntariamente, excluyéndose aquellos que, habiendo sido seleccionados, rehusaron participar $(n=18)$.

La muestra está dividida en tres grupos de edad. Las características demográficas de la muestra, para cada uno de los intervalos de edad, se muestran en la Tabla 1.

Tabla 1.- Características demográficas de la muestra

\begin{tabular}{|ccccccc|}
\hline $\begin{array}{l}\text { Intervalo } \\
\text { de edad } \\
\text { (anos) }\end{array}$ & $\mathrm{n}$ & $\begin{array}{l}\text { Media } \\
\text { edad } \\
\text { (años) }\end{array}$ & $\begin{array}{c}\% \\
\text { mujeres }\end{array}$ & $\begin{array}{l}\% \\
\text { sin } \\
\text { estudios }\end{array}$ & $\begin{array}{c}\% \\
\text { estudios } \\
\text { primarios }\end{array}$ & $\begin{array}{c}\% \\
\text { residencia }\end{array}$ \\
\hline$<70$ & 56 & 64.96 & 58.9 & 35.7 & 53.6 & 18.2 \\
$70-79$ & 88 & 74.68 & 53.4 & 48.9 & 46.6 & 43.2 \\
$>80$ & 86 & 84.44 & 60.5 & 57.6 & 35.3 & 62.4 \\
TOTAL & 230 & 75.97 & 57.4 & 48.9 & 44.1 & 44.3 \\
\hline
\end{tabular}


De acuerdo con las características sociodemográficas de la población española mayor de 65 años, se observa que el número de mujeres es superior al de hombres en todos los intervalos de edad y que la inmensa mayoría no tiene estudios o sólo tiene estudios primarios. En la muestra se comprueba también una mayor proporción de sujetos que viven en la residencia a medida que aumenta la edad.

La recogida de datos se realizó durante el año 1995 en tres lugares: Residencia de Válidos del INSERSO, Hogares del Pensionista de la ciudad y domicilios particulares. Lo que nos ha permitido establecer dos grupos: 1) los que viven en residencia, procedentes del primer ambiente; y 2) los que viven en su propio domicilio. Los datos se obtuvieron mediante una entrevista personal de unos 90 minutos de duración, que es el tiempo que se tarda en administrar el conjunto de todas las pruebas.

Al pase de los cuestionarios nos ayudaron cinco alumnos de un curso de "Evaluación e Intervención Psicológica en la Vejez", organizado por el Departamento de Psicología, a los que se les entrenó para el manejo de las distintas escalas de evaluación. Las sesiones de entrenamiento de los alumnos consistieron en: 1) Tres sesiones, con un total de diez horas, de conocimiento y manejo de los cuestionarios que se iban a utilizar. En estas sesiones se incluyó la visión de un vídeo en el que se pasaba el MMSE y otras pruebas a un anciano; 2) El pase de dos pruebas a otros participantes en el curso, a modo de entrenamiento; y 3) La presencia, de uno en uno, en el pase real de los cuestionarios, realizado por los investigadores, a dos ancianos. Una vez formados, a los alumnos se les asignó aleatoriamente una media de cuarenta sujetos de la lista de ancianos de los que se disponía para la investigación, estando acompañados por los investigadores en sus dos primeras entrevistas reales. Con el fin de evaluar el grado de coincidencia en el regis-tro, de las distintas puntuaciones de las pruebas utilizadas, se hizo un analisis de varian-za de la puntuaciones del MMSE $(F(5,224)=1.28, p=.270)$ y del CES-D $(F(5,208)$ $=2.17, p=.058$ ), no encontrándose diferencias sig-nificativas interjueces en ninguna de las dos pruebas.

\section{Medidas}

Se han utilizado dos escalas de depresión: CES-D y ESCALA DE DEPRESION según criterios del DSM III-R, que se han pasado conjuntamente con otras pruebas sobre deterioro cognitivo (Entrevista Neuropsicológica, Tests neuropsicológicos) y con un cuestionario socio-demográfico.

La versión española utilizada de la escala CES-D es de carácter experimental, ya que en estos momentos nos encontramos en fase de adaptación psicométrica de la misma a nuestro entorno, en una muestra representativa de la población de mayores de 20 años. Los datos provisionales $(n=497)$ de que disponemos, fruto de otro proyecto de investigación que se está realizando en el departamento, nos muestran que la escala presenta un alto índice de consistencia interna $(\alpha=.916)$. En la muestra de ancianos, utilizada para este trabajo, el indice de consistencia interna es también elevado $(\alpha=.849)$.

La escala CES-D consta de 20 items que representan otros tantos sintomas del desorden depresivo (ver Tabla 2). Los entrevistados son instados a sefialar la frecuencia en la que han experimentado cada sintoma durante la semana anterior al momento de la entrevista. Las respuestas varian desde "ninguna vez" (0) hasta "muchas veces" (3). Cuatro fterns $(4,8$, 12 y 16) tienen un significado positivo respecto a la depresión y se puntúan en consecuencia de forma inversa. La puntuación total del CES$D$ es la suma de todos los items con un rango de puntuaciones posibles entre 0 y 60 . Una puntuación de 16 o más se ha utilizado para clasificar a las personas que tienen "síntomas depresivos". El punto de corte ha sido validado con los criterios de depresión clínica del DSMIII-R (Comstock y Helsing, 1976; Radloff, 1977; Eaton y Kessler, 1981; Frerichs, Aneshensel y Clark, 1981; Berkman et al., 1986; Andresen, Malmgren, Carter y Patrick, 1994) y ha sido usado con distintas poblaciones (Myers y Weissman, 1980; Boyd, Weissman, Thompson y Myers, 1982; Hankin y Locke, 1982; Roberts y Vernon, 1983; Kennedy, Kelman, Thomas, Wisniewski, Metz y Bijur, 1989; Costa, McCrae y Locke, 1990; Brown, Milburn y Gary, 1992). Algunos autores han usado puntos de corte más altos (Himmelfarb, Murrel, 1983; Murrel et al., 1983) y otros han optado por usar como 
Tabla 2.- Items de la Escala de CES-D (adaptación española)

\begin{tabular}{|rl|}
\hline Item $n^{\circ}$ & \multicolumn{1}{c|}{ Item } \\
\hline 3 & \multicolumn{1}{c|}{ Estado de ánimo deprimido } \\
6 & Me sentia triste y descorazonado, a pesar de la ayuda de mi familia y amigos \\
9 & Me sentia deprimido \\
10 & Pensaba que mi vida habia sido un fracaso \\
14 & Me sentia atemorizado \\
17 & Me sentia solo \\
18 & Hubo ratos en que lloré \\
& Me sentia triste \\
& \\
1 & Me molestaron cosas que normalmente no me molestan \\
2 & No tenia hambre, no me apetecia comer \\
5 & Tenia dificultad para concentrarme \\
7 & Para hacer cualquier cosa, tenia que hacer un gran esfuerzo \\
11 & No me sentia descansado, después de haber dormido \\
13 & Hablaba menos de lo habitual \\
20 & Me encontraba sin fuerzas para hacer nada \\
& \\
15 &
\end{tabular}

punto de corte la media de la puntuación total (Myers y Weisman, 1980; Rodriguez-Narajo y Godoy, 1997). Nosotros hemos preferido mantener el punto de corte más avalado por la bibliografia al uso y que nos permite comparar nuestros resultados con los de otros trabajos similares.

Los diversos estudios, que han utilizado el análisis factorial para encontrar subescalas en el CES-D (Hertzog, 1989; Hertzog, Van Alstine, Usala, Hultsch y Dixon, 1990), coinciden en que: a) hay 4 factores más o menos equivalentes en las distintas etapas del desarrollo adulto, $y$ b) existe un factor de segundo orden de depresión. Los 4 factores se denominan: "Estado de ánimo deprimido o afecto negativo", "sintomas somáticos o retardo psicomotor", "afecto positivo o bienestar" (actualmente "ausencia de bienestar" ya que se puntúa de forma inversa), y "dificultades interpersonales". El factor de segundo orden es un factor general de depresión. En el trabajo de adaptación de la escala a nuestro entorno, que paralelamente estamos realizando a éste, aparecen los mismos cuatro factores, que explican un $60,8 \%$ de la varianza. La fiabilidad de las subescalas en la muestra total es de $.89, .76, .78$ y .67 para estado de ánimo deprimido, retardo psicomotor, ausencia de bienestar y dificultades interpersonales respectivamente.

La Escala de Depresión según criterios del DSM-III-R para el diagnóstico clínico de un 
Tabla 3.- Escala de Depresión según criterios DSM-III-R (Episodio Depresivo Mayor)

¿Podría decirme si en el pasado ano ha sentido al menos durante dos semanas las siguientes sensaciones?

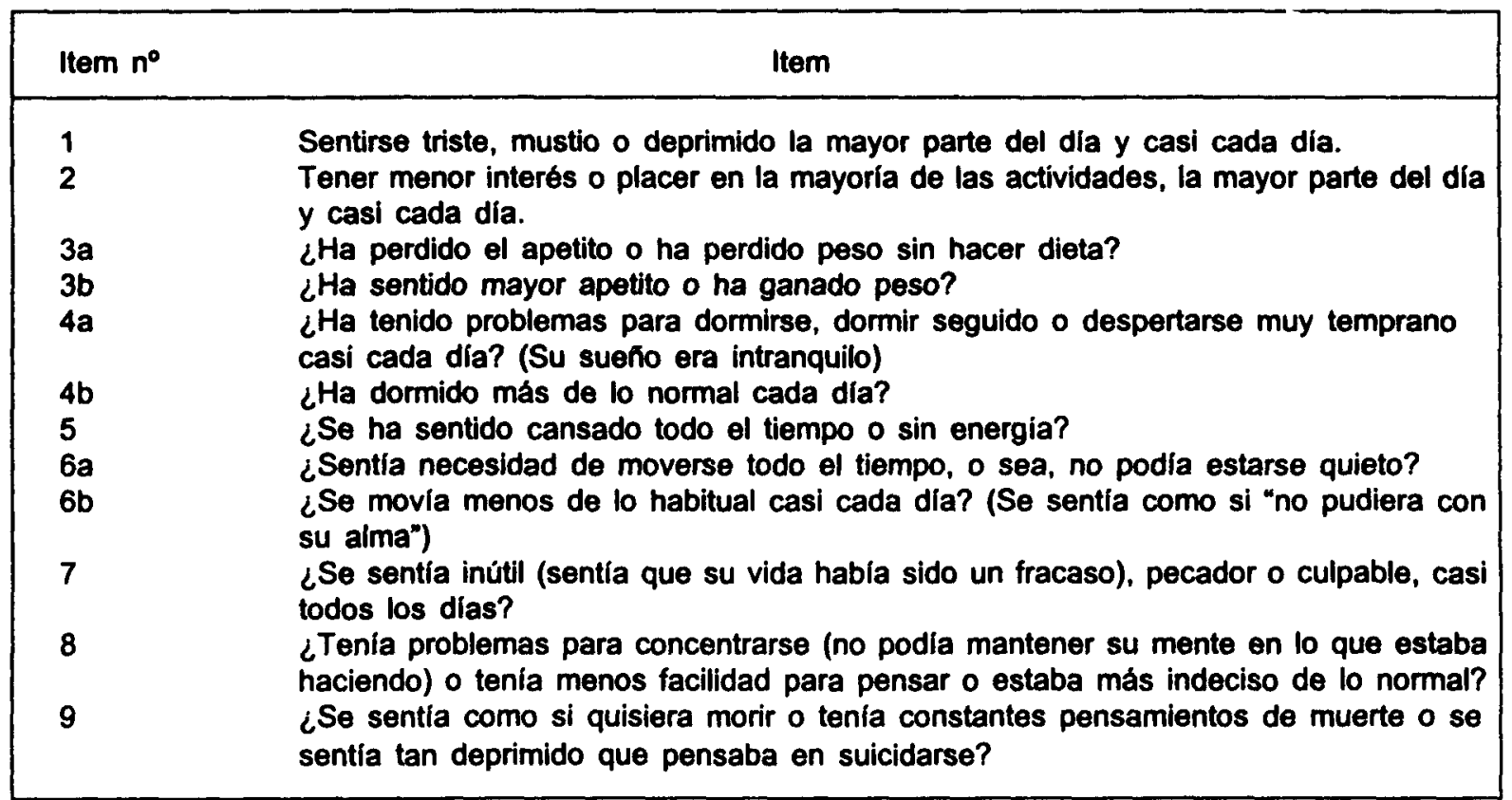

Episodio Depresivo Mayor (EDM) consta de 9 items, de los que 3 se desdoblan (ver Tabla 3). Esta escala permite evaluar si el sindrome depresivo es "nulo", "cuestionable" o "presente". Se considera que el sindrome depresivo está "presente" si al menos cinco sintomas (items) se confirman en el sujeto, y uno de los cinco es el primero o el segundo de la tabla. En el caso de que se confirmen 5 sintomas o más, pero ninguno sea el primero o el segundo, se evalúa la depresión como "cuestionable".

Para la evaluación del deterioro cognitivo hemos adaptado la Batería de Test para la Demencia, similar a la utilizada en un estudio sobre la demencia realizado en Suecia (Pedersen, Gatz, Winblad, Pearson y Berg, 1988). La bateria consta de Entrevista neuropsicológica y Tests neuropsicológicos. Además, dentro de la entrevista neuropsicológica se incorporan los items de la escala de deterioro cognitivo Mini Mental State Examination (MMSE, Folstein, Folstein y McHugh, 1975). Esta escala puntúa de 0 a 30, considerándose una puntuación de corte para el deterioro cognitivo el 23. EI MMSE es una herramienta excelente y ampliamente utilizada para la evaluación del deterioro cog- nitivo, como lo demuestra el que si se comparan las relaciones entre la Escala de deterioro global (GDS), un indice clínico ampliamente utilizado para el diagnóstico del deterioro, y diferentes medidas comportamentales y biologicas, la mayor correlación se da con el MMSE (.89) (Reisberg, Ferris, Steinberg, Shulman, de León y Sinaiko, 1989).

La entrevista neuropsicológica consta de las siguientes pruebas: 1) Orientación (conocimiento del lugar geográfico donde vive, del día, mes, año actuales, etc.); 2) Metamemoria (percepción de problemas de memoria como olvidar nombres de amigos, articulos que comprar, compromisos sociales, etc.); 3) Memoria y control mental (recordar nombres de objetos inmediatamente después de ser presentados y después de una tarea distractora, restar hacia atrás, etc.); 4) Lenguaje (nombrar objetos y dibujos, ejecutar mandatos verbales, hacer, repetir e interpretar frases, etc.); 5) Juicio y abstracción (establecer semejanzas y diferencias, realizar operaciones matemáticas con monedas, etc.); y 6) Funcionamiento visoespacial (copiar figuras geométricas, dibujar un reloj señalando distintas horas, etc.). 
Los tests neuropsicológicos, desarrollados por el CERAD (Morris, Mohs, Rogers, Fillenbaum y Heyman, 1988), y ampliamente utilizados en EE.UU., comprenden: 1) Fluidez verbal (nombrar animales durante un minuto); 2) Memoria y aprendizaje (aprendizaje y recuerdo inmediato de una lista de palabras, con tres ensayos en los que varia el orden de presentación); 3) Recuerdo demorado (recordar lista de palabras anterior después de 5 minutos); 4) Reconocimiento (tarea de reconocer las palabras de la lista anterior, confundidas con otras no presentadas); y 5) Memoria lógica (recuerdo de una historia).

El conjunto de pruebas neuropsicológicas muestra una consistencia alta $(\alpha=.87)$. El análisis factorial nos da dos factores claramente diferenciados. El primero incluye las tareas de fluidez verbal, lenguaje, funcionamiento visoespacial, juicio y abstracción, memoria lógica, orientación, memoria, lista de palabras y recuerdo demorado ( $48.8 \%$ de varianza). El segundo incluye las tareas de reconocimiento y el autoinforme sobre la metamemoria $19.9 \%$ de varianza). El primer factor correlaciona con la edad (-.39), con la puntuación del MMSE (.82) y con la puntuación en la escala CES-D $(-.29)$. El segundo factor correlaciona con la puntuación del MMSE (.31), con la puntuación en la escala CES-D (-.23), pero no con la edad.

Todas estas pruebas las hemos completado con un cuestionario sobre datos sociodemográficos en los que se incluye la edad, el género, el lugar de residencia y el nivel de estudios de los sujetos de la muestra.

\section{RESULTADOS}

\section{Edad y género}

La primera cuestión que hemos pretendido evaluar es si existen diferencias en la sintomatologia depresiva, medida por las dos escalas, en función de la edad y del género de la muestra. Para ello se han analizado los resultados de la escala CES-D de tres formas: a) comparación de medias y ANOVA, b) comparación de las dos escalas en la estimación de la prevalencia de la sintomatología depresiva, y c) evaluación combinada con el MMSE para diferenciar depresión y deterioro cognitivo. Estas medidas, a su vez, se han comparado, en algunos casos, con la distribu- ción porcentual del sindrome depresivo según el criterio del DSM-III-R.

\section{a) Análisis de las puntuaciones medias y ANOVA on la escala CES-D}

Las puntuaciones medias, el ANOVA de una via y los contrastes, tanto ortogonales como a priori, para el total y las distintas subescalas del CES-D por edad se pueden ver en la tabla 4.

Comparando las puntuaciones medias para cada una de las subescalas y la puntuación media total podemos ver que existen diferencias significativas entre los grupos de edad en el total y en la subescala "ánimo deprimido", para los contrastes ortogonales utilizados. Cuando se contrasta cada uno de los grupos con el resto, se aprecia que los grupos 2 y 3 se diferencian de forma significativa con el resto. Los resultados totales ofrecen una curva en forma de $U$, sin embargo, como se puede ver en la gráfica 1, en el grupo de los hombres, además de que las puntuaciones son inferiores en todos los grupos de edad, la curva se convierte en una línea ascendente.

Si hacemos un ANOVA de dos vias (edad y sexo) para la puntuación total se siguen manteniendo las diferencias significativas en función de la edad, $F(2,208)=3.34, p<.01, y$, además, en función del género, $F(1,208)=8,77$, $p<.01$. Las mujeres presentan una puntuación mayor en todos los grupos de edad, no existiendo efecto de interacción edad-sexo.

b) Comparación de las dos escalas en la estimación de la prevalencla de la sintomatologia depresiva

Para evaluar la prevalencia de la sintomatologia depresiva hemos utilizado, como ya deciamos anteriormente, la puntuación de corte del CES-D (mayor o igual a 16) y la evaluación del sindrome depresivo según criterios DSM-III-R. En la tabla 5, se muestran los porcentajes de ambas pruebas, para cada una de las edades y sexo. En la columna de prevalencia según CES-D aparece el porcentaje de sujetos que han obtenido una puntuación de 16 o más, mientras que en la columna de pre-valencia según el DSM-III-R se especifica sólo el porcentaje de sujetos que se incluyen en el criterio "sindrome presente". 
Tabla 4.- Comparación de medias y ANOVA para las subescalas y el total del CES-D, en función de los grupos de edad, incluyendo los contrastes ortogonales y los contrastes a priori

\begin{tabular}{|c|c|c|c|c|c|}
\hline \multirow{2}{*}{ VARIABLE } & \multicolumn{5}{|c|}{ SUBESCALA } \\
\hline & CES-D & $A D$ & RS & $A B$ & $\mathrm{DI}$ \\
\hline $\begin{array}{l}\text { GRUPOS DE } \\
\text { EDAD }\end{array}$ & \multicolumn{5}{|c|}{ Comparaciones entre grupos de edad } \\
\hline $\begin{array}{l}\text { (1) < } 70 \\
\text { media } \\
\text { des.est. }\end{array}$ & $\begin{array}{l}14.52 \\
9.84\end{array}$ & $\begin{array}{l}4.20 \\
4.26\end{array}$ & $\begin{array}{l}4.48 \\
4.35\end{array}$ & $\begin{array}{l}5.26 \\
3.42\end{array}$ & $\begin{array}{l}0.65 \\
1.09\end{array}$ \\
\hline $\begin{array}{l}\text { (2) } 70-79 \\
\text { media } \\
\text { des.est. }\end{array}$ & $\begin{array}{r}13.01 \\
9.44\end{array}$ & $\begin{array}{l}3.41 \\
4.13\end{array}$ & $\begin{array}{l}4.13 \\
4.09\end{array}$ & $\begin{array}{l}5.33 \\
3.39\end{array}$ & $\begin{array}{l}0.65 \\
1.12\end{array}$ \\
\hline $\begin{array}{l}\text { (3) > } 80 \\
\text { media } \\
\text { des.est. }\end{array}$ & $\begin{array}{l}17.46 \\
11.09\end{array}$ & $\begin{array}{l}5.30 \\
4.96\end{array}$ & $\begin{array}{l}5.22 \\
4.53\end{array}$ & $\begin{array}{l}5.96 \\
3.26\end{array}$ & $\begin{array}{l}0.79 \\
1.40\end{array}$ \\
\hline $\begin{array}{l}\text { TOTAL } \\
\text { media } \\
\text { des.est. }\end{array}$ & $\begin{array}{l}15.03 \\
10.31\end{array}$ & $\begin{array}{l}4.31 \\
4.54\end{array}$ & $\begin{array}{l}4.61 \\
4.33\end{array}$ & $\begin{array}{l}5.55 \\
3.35\end{array}$ & $\begin{array}{l}0.70 \\
1.22\end{array}$ \\
\hline Mce & 103.56 & 20.18 & 18.70 & 11.25 & 1.50 \\
\hline $\mathbf{F}$ & $3.94^{*}$ & $3.69^{*}$ & 1.34 & 0.99 & 0.32 \\
\hline \multirow{2}{*}{ F Lineal } & \multicolumn{5}{|c|}{ Contrastes ortogonales } \\
\hline & $4.28^{*}$ & $4.52^{*}$ & 1.40 & 0.35 & 0.16 \\
\hline \multirow{3}{*}{$\begin{array}{l}\text { t } \\
\text { Grupo } 2 \\
\text { vs } 1,3 \\
\text { Grupo } 3 \\
\text { vs } 1,2\end{array}$} & \multicolumn{5}{|c|}{ Otros contrastes } \\
\hline & $-2.07^{*}$ & $-2.12^{*}$ & -1.18 & -0.59 & -0.40 \\
\hline & $2.54^{*}$ & $2.36^{*}$ & 1.49 & 1.41 & 0.80 \\
\hline
\end{tabular}

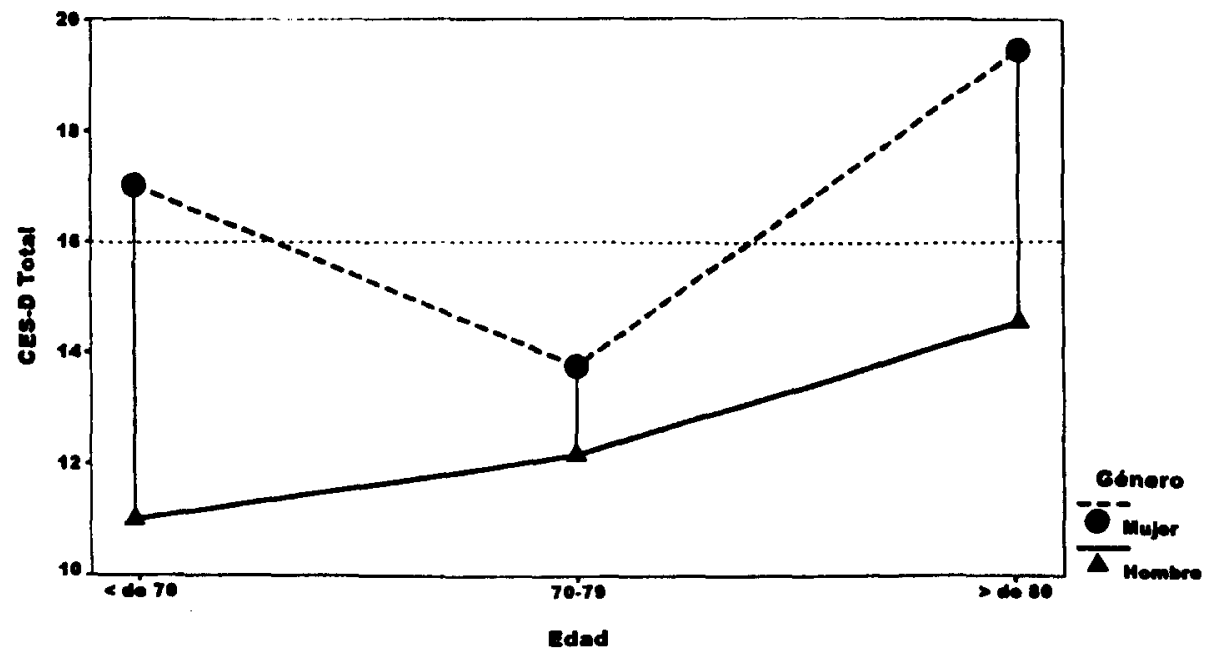

Figura 1.- Depresión por edad y género 
Tabla 5.- Prevalencia de la depresión, comparando las dos escalas

\begin{tabular}{|c|c|c|c|c|c|c|}
\hline \multirow{3}{*}{ EDAD } & \multicolumn{6}{|c|}{ PREVALENCIA DEPRESION } \\
\hline & \multicolumn{2}{|c|}{ TOTAL } & \multicolumn{2}{|c|}{ HOMBRES } & \multicolumn{2}{|c|}{ MUJERES } \\
\hline & CES-D & DSM-III-R & CES-D & DSM-III-R & CES-D & DSM-III-R \\
\hline $\begin{array}{l}<70 \\
70-79 \\
>80\end{array}$ & $\begin{array}{l}32.1 \\
35.4 \\
44.3\end{array}$ & $\begin{array}{l}35.7 \\
35.2 \\
41.9\end{array}$ & $\begin{array}{l}22.7 \\
33.3 \\
25.0\end{array}$ & $\begin{array}{l}21.7 \\
29.3 \\
26.5\end{array}$ & $\begin{array}{l}38.7 \\
37.2 \\
57.4\end{array}$ & $\begin{array}{l}45.5 \\
40.4 \\
51.9\end{array}$ \\
\hline TOTAL & 37.9 & 37.8 & 28.0 & 26.5 & 45.5 & 46.2 \\
\hline
\end{tabular}

La prevalencia de la depresión para el total de la muestra es prácticamente igual según el CES-D (37.9) y según el criterio DSM-III-R (37.8). Esta prevalencia es significativamente mayor en las mujeres (45.5 6 46.2) que en los hombres (28.0 o 26.5). Por edades, los porcentajes más altos de depresión se dan en los mayores de 80 años, para el total de la muestra y para las mujeres. Los hombres presentan los porcentajes más altos entre los 70 y los 80 años.

Nos encontramos, por tanto, con una gran semejanza en la estimación de la depresión por las dos escalas, en la muestra total y en las muestras por género. Si se cruzan los porcentajes de ambas escalas se observa un $68.7 \%$ de coincidencia y un $16.8 \%$ de discrepancia, confirmándose la semejanza en la estimación de la depresión entre las dos escalas $\left(\chi^{2}=67.3, p=.00 ; C=.49, p=.00\right)$. Además, los evaluados como "cuestionables" por la escala clínica DSM-III-R se distribuyen por igual entre sindrome "presente" (7.0\%) y "no presente" $(7.5 \%)$ del CES-D, lo cual reafirma la semejanza en la evaluación total de ambas escalas, cuando se utiliza como punto de corte una puntuación de 16 . En nuestros datos una puntuación de corte más alta, como la utilizada en algunos trabajos (v.g. 20), supone una disminución de la coincidencia de ambas estimaciones. Asl, el porcentaje de coincidencia se reduce a un $63.6 \%$ en el sindrome presente y aumenta la discrepancia a un $21.9 \%$, aunque la relación entre las dos escalas sigue siendo significativa $\left(\chi^{2}=44.3, p .=.00 ; C=.41, p=.00\right)$.

\section{c) Evaluación diferencial de depresión y deterioro cognitivo}

Los resultados, anteriormente expuestos, varian si tenemos en cuenta la posible confu- sión entre depresión y deterioro cognitivo que se puede dar en la vejez, como apuntábamos en la introducción. En efecto, si utilizamos como grupos de comparación los tipos de diagnóstico siguientes: ancianos normales, ancianos con depresión sin deterioro cognitivo, ancianos con deterioro cognitivo sin depresión y ancianos con deterioro cognitivo y depresión, los resultados muestran una tendencia distinta a la que hemos visto hasta ahora (Tabla 6). Mientras que la prevalencia del deterioro cognitivo (sin depresión) se incrementa con la edad, la prevalencia de la depresión (sin deterioro cognitivo) disminuye. El porcentaje medio de depresión (sin deterioro) disminuye hasta el $10.7 \%$, cuando en los resultados anteriores el porcentaje de depresión total (incluyendo deterioro), alcanzaba un $37.9 \%$, porcentajes que están más de acuerdo con la literatura existente sobre el tema.

Si en el análisis de las puntuaciones medias y ANOVA, obtenidas con la escala CES$D$, hemos visto que en la puntuación total hay diferencias significativas en función de la edad $(F(2,211)=3.94, p=.02)$, cuando se excluyen del cálculo los sujetos que presentan deterioro cognitivo (punto de corte en MMSE para una puntuación menor o igual a 23), y nos quedamos solamente con 101 sujetos de la muestra total, desaparecen las diferencias significativas en función de la edad $(F(2,98)=0.18, p=.83)$. Además se reduce la media en todas las subescalas del CES-D (AD $=2.81, R S=3.12$, $A B=4.69$ y $\mathrm{Dl}=0.55)$ y en el total $(11.18)$ en todos los intervalos de edad.

\section{Lugar de residencia}

Las personas ancianas, que se ven obligadas a salir de su entorno familiar y viven en una Residencia, presentan una prevalencia mayor 
Tabla 6.- Prevalencia de la depresión y el deterioro cognitivo, por edades

\begin{tabular}{|lllll|}
\hline EDAD & DEPRESION & DETERIORO & CONJUNTO & AUSENCIA \\
\hline$<70$ & 13,2 & 13,2 & 18,9 & 54,7 \\
$70-79$ & 13,4 & 25,6 & 22,0 & 39,0 \\
$>80$ & 6,3 & 34,2 & 34,2 & 38,0 \\
TOTAL & 10,7 & 25,7 & 27,1 & 36,4 \\
\hline
\end{tabular}

Tabla 7.- Prevalencia de la depresión por lugar de residencia

\begin{tabular}{|l|llllll|}
\hline & \multicolumn{4}{|c}{ RESIDENCIA } & \multicolumn{3}{c|}{ DOMICILIO PROPIO } \\
\cline { 2 - 7 } ESCALA & TOTAL & HOMBRE & MUJER & TOTAL & HOMBRE & MUJER \\
\hline CES-D & 40.9 & 34.9 & 46.7 & 35.2 & 22.0 & 44.0 \\
DSM-III-R & 40.6 & 29.8 & 50.0 & 35.4 & 23.5 & 43.4 \\
& & & & & & \\
\hline
\end{tabular}

de depresión que las que siguen viviendo en su propio domicilio (Tabla 7). No obstante, estas diferencias no son estadísticamente significativas. Las dos pruebas confirman unos porcentajes más elevados de depresión en las mujeres que en los hombres, aunque la diferencia de prevalencia, medida por el CES-D, es mayor entre hombres que viven en el domicilio propio y hombres que viven en la residencia que entre las mujeres de ambos lugares.

Con respecto a la comparación de medias de las subescalas del CES-D, entre los residentes y no residentes, tampoco se han encontrado diferencias significativas ni en el total ni en las cuatro subescalas.

\section{Nivel de estudios}

La variable nivel de estudios, que se ha dividido en tres categorias (sin estudios, estudios primarios y estudios secundarios o más), se relaciona inversamente con la depresión, de forma que a mayor nivel de estudios menor prevalencia de la depresión $\left(\chi^{2}=7.42\right.$ p. $\left.=.02\right)$ y menor puntuación total del CES-D ( $F(2,211)$ $=3.86, p=.02$ ).

Esta relación está mediatizada por la interacción existente entre el nivel de estudios y el deterioro cognitivo. Si efectuamos un análisis de la covarianza (ANCOVA), utilizando como covariada la puntuación de deterioro cognitivo
(MMSE), el efecto del nivel de estudios sobre la depresión desaparece $(F(2,210)=0.88, p=.41)$ y resulta significativo el efecto de la covariada $(F(1,210)=30.86, p=.00)$.

\section{Depresión, variables cognitivas y nivel de estudios}

Si comparamos mediante un ANOVA los cuatro grupos de sujetos anteriormente sefialados (normales, con depresión sin deterioro cognitivo, con deterioro cognitivo sin depresión y con deterioro cognitivo y depresión) en la ejecución de los distintos tests neuropsicologicos y la metamemoria, encontramos que todos ellos producen diferencias significativas entre los cuatro grupos, siendo las más importantes las ocasionadas por la fluidez verbal, memoria lógica y recuerdo inmediato (tabla 8). Esto nos va a permitir un mayor acercamiento al diagnóstico diferencial de la depresión a partir de estas pruebas.

Como se puede ver en los contrastes a priori, la tarea de metamemoria es la que mejor discrimina entre sujetos normales y deprimidos sin deterioro. Mientras que todas las tareas, destacando la fluidez verbal y el recuerdo inmediato, discriminan a los sujetos normales de los sujetos con deterioro cognitivo, ya que las diferencias son siempre significativas. En la comparación entre ancianos con deterioro cognitivo 
Tabla 8.- Comparación de medias y ANOVA para los cuatro grupos de diagnóstico, incluyendo los contrastes a priori

\begin{tabular}{|c|c|c|c|c|c|}
\hline Prueba & $\begin{array}{l}\text { Normales } \\
(n=78)\end{array}$ & $\begin{array}{l}\text { Depresión } \\
(n=23)\end{array}$ & $\begin{array}{l}\text { Deterioro } \\
(n=55)\end{array}$ & $\begin{array}{l}\text { Ambos } \\
(n=58)\end{array}$ & $F(3,210)^{a}$ \\
\hline $\begin{array}{l}\text { Metamemoria } \\
\text { Fluidez Verbal } \\
\text { (4.14) } \\
\text { Recuerdo Inmd. } \\
\text { Lista palabras } \\
\text { Recuerdo } \\
\text { demorado } \\
\text { Reconocimiento } \\
\text { Memoria lógica }\end{array}$ & $\begin{array}{l}5.09^{\circ} \\
(1.29)^{\circ} \\
15.62 \\
(4.24) \\
5.05 \\
(1.46) \\
4.59 \\
(2.33) \\
17.86 \\
(3.18 \\
10.80 \\
(3.66)\end{array}$ & $\begin{array}{l}4.30 \\
(1.49) \\
13.78 \\
(4.43) \\
4.72 \\
(1.56) \\
4.61 \\
(2.19) \\
16.74 \\
(4.30) \\
9.87 \\
(3.84)\end{array}$ & $\begin{array}{l}4.11 \\
(1.67) \\
10.87 \\
(3.82) \\
3.53 \\
(1.69) \\
2.86 \\
(2.12) \\
15.46 \\
(5.15) \\
7.60 \\
(3.90)\end{array}$ & $\begin{array}{l}3.52 \\
(1.98) \\
9.50 \\
(.000) \\
3.21 \\
(1.52) \\
2.55 \\
(2.07) \\
15.10 \\
(4.60) \\
5.94 \\
(3.67)\end{array}$ & $\begin{array}{l}10.93^{c} \\
(.000)^{\circ} \\
28.34 \\
19.10 \\
(.000) \\
12.15 \\
(.000) \\
5.49 \\
(.001) \\
19.95 \\
(.000)\end{array}$ \\
\hline \multicolumn{6}{|c|}{$\begin{array}{l}a=\text { media } \\
b=\text { desviación típica } \\
c=\text { valor de } F \\
d=\text { nivel de significación } \\
\text { a } p<0,001 \text { en todos los casos }\end{array}$} \\
\hline & NC-DP & NC-DC & DP-DC & DP-AM & DC-AM \\
\hline $\begin{array}{l}\text { Metamemoria } \\
\text { Fluidez verbal } \\
\text { Recuerdo inmd. } \\
\text { Lista palabras } \\
\text { Recuerdo retd. } \\
\text { Reconocimiento } \\
\text { Memoria Iógica }\end{array}$ & $\begin{array}{l}2.287^{* *} \\
1.831^{* *} \\
\text { NS } \\
\text { NS } \\
\text { NS } \\
\text { NS }\end{array}$ & $\begin{array}{l}3.646^{* *} \\
6.241^{* *} \\
5.325^{* *} \\
4.330^{* *} \\
2.992^{* *} \\
4.682^{* *}\end{array}$ & $\begin{array}{l}\text { NS } \\
2.826^{*} \\
3.097^{* *} \\
3.157^{* *} \\
\text { NS } \\
2.425^{*}\end{array}$ & $\begin{array}{l}\text { NS } \\
4.211^{* *} \\
3.902^{* *} \\
3.754^{* *} \\
\text { NS } \\
4.159^{* *}\end{array}$ & $\begin{array}{l}\text { NS } \\
\text { NS } \\
\text { NS } \\
\text { NS } \\
\text { NS } \\
2.244^{*}\end{array}$ \\
\hline \multicolumn{6}{|c|}{$\begin{array}{l}\text { NC: Normales; DP: Con depresión; DC: Con deterioro cognitivo; } \\
\text { AM: Con depresión y deterioro cognitivo } \\
p=.05, * p=.01\end{array}$} \\
\hline
\end{tabular}

y ancianos con depresión, las diferencias son significativas en todos los casos, excepto en metamemoria y reconocimiento, destacando el poder discriminativo de la prueba de recuerdo demorado. Por último, a la hora de distinguir entre la depresión y el diagnóstico conjunto (depresión-deterioro), además del recuerdo demorado, los tests de memoria lógica y fluidez verbal poseen un alto valor discriminativo.

También hemos realizado una análisis discriminante con el fin de encontrar qué tareas cognitivas discriminan mejor entre sujetos de- primidos y no deprimidos, asi como entre sujetos con deterioro cognitivo y sin él en relación con el nivel de estudios, dada la influencia de éste sobre el deterioro cognitivo $y$, en menor medida, sobre la depresión (tabla 9). Como se puede ver, a través de los coeficientes de correlación canónica, el conjunto de pruebas utilizadas obtiene una mejor función discriminante en el caso del deterioro cognitivo que en el de la depresión.

Fluidez verbal y metamemoria discriminan mejor la depresión, mientras que memoria lógi- 
Tabla 9.- Comparación de medias y ANOVA para los cuatro grupos de diagnóstico, incluyendo los contrastes a priori

1) ustodo: Indepandientee Juntae

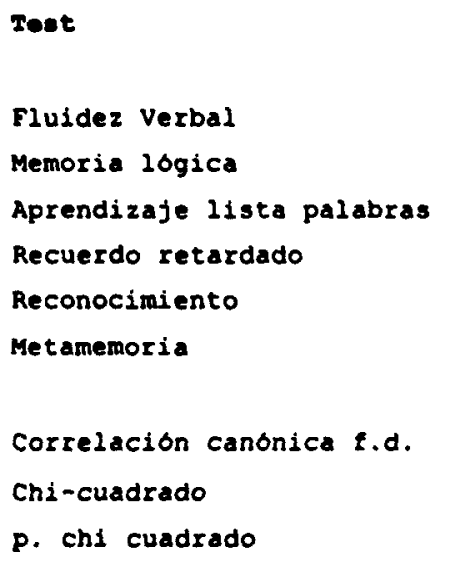

2) Whtodo: Inclueión por pereos

\begin{tabular}{|c|c|c|c|c|c|}
\hline \multicolumn{3}{|c|}{ Depreason } & \multicolumn{3}{|c|}{ Detarioro } \\
\hline Total & 82 & 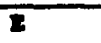 & Total & 8I & $\bar{E}$ \\
\hline .76 & .53 & .76 & .64 & .64 & .67 \\
\hline .69 & .95 & .38 & .77 & .81 & .63 \\
\hline .47 & .42 & .36 & .70 & .71 & .65 \\
\hline .32 & .33 & .21 & .59 & .58 & .61 \\
\hline .30 & .21 & .24 & .47 & .41 & .45 \\
\hline .64 & .37 & .70 & .34 & .23 &, 51 \\
\hline .36 & .30 & .47 & .61 & .59 & .56 \\
\hline 26,39 & 7,71 & 23,71 & 93,15 & 39,32 & 39,21 \\
\hline .000 & n.s. & .000 & .000 & .000 & .000 \\
\hline
\end{tabular}

Toet

Fluidez Verbal
Memoria logica
Aprendizaje lista palabras
Recuerdo retardado
Reconocimiento
Metamemoria

Correlación canónica $\mathbf{t} . \mathrm{d}$.

Chi-cuadrado

p. chi cuadrado

\begin{tabular}{|c|c|c|c|c|c|}
\hline \multicolumn{3}{|c|}{ Depren16n } & \multicolumn{3}{|c|}{ Doterioro } \\
\hline Total & 8 & $\mathbf{E}$ & Total & 8E & 8 \\
\hline 81 & .48 & .79 & .66 & .48 & .73 \\
\hline .45 & 1,00 & .42 & 28 & 88 & $\angle 70$ \\
\hline .52 & .50 & .49 & .72 & 77 & .45 \\
\hline .42 & .37 & .40 & .53 & .53 & .39 \\
\hline .24 & .22 & .09 & 48 & .23 & 50 \\
\hline .69 & .13 & .73 & .16 & .01 & .23 \\
\hline .34 &, 28 & .45 &, 60 &, 55 & .52 \\
\hline 23,60 & 7,26 & 22,35 & 90,35 & 34,86 & 33,81 \\
\hline .000 &, 007 & .000 & .000 & .000 & .000 \\
\hline
\end{tabular}

Nota: SE: sin estudios; E: estudios primarios o mas. Se muestran las correlaciones de cada test con la función discriminante $y$, en el mótodo de inclusión por pasos se subrayan los coeficientes de las variables que se incluyen en el andlisis.

ca, recuerdo inmediato y fluidez verbal discriminan mejor el deterioro cognitivo. Si tenemos en cuenta el nivel de estudios, las funciones discriminantes varian de forma significativa, sobre todo en el caso de la depresión. Asi, mediante el método de inclusión por pasos, la única variable que resulta discriminativa de la depresión en sujetos sin estudios es la memoria lógica. Sin embargo, en los sujetos con estudios, las variables que mejor discriminan son la fluidez verbal y la metamemoria.

\section{Interrelación entre las variables estudiadas}

A lo largo de la exposición precedente de resultados, se constata una especial interrelación entre depresión y las variables estudiadas. En la matriz de correlaciones (Tabla 10) se puede ver de forma resumida estas interrelaciones.

La depresión aparece relacionada con todas las variables de deterioro cognitivo (pruebas cognitivas), con la puntuación del MMSE, con el género y con el nivel de estudios. Las 


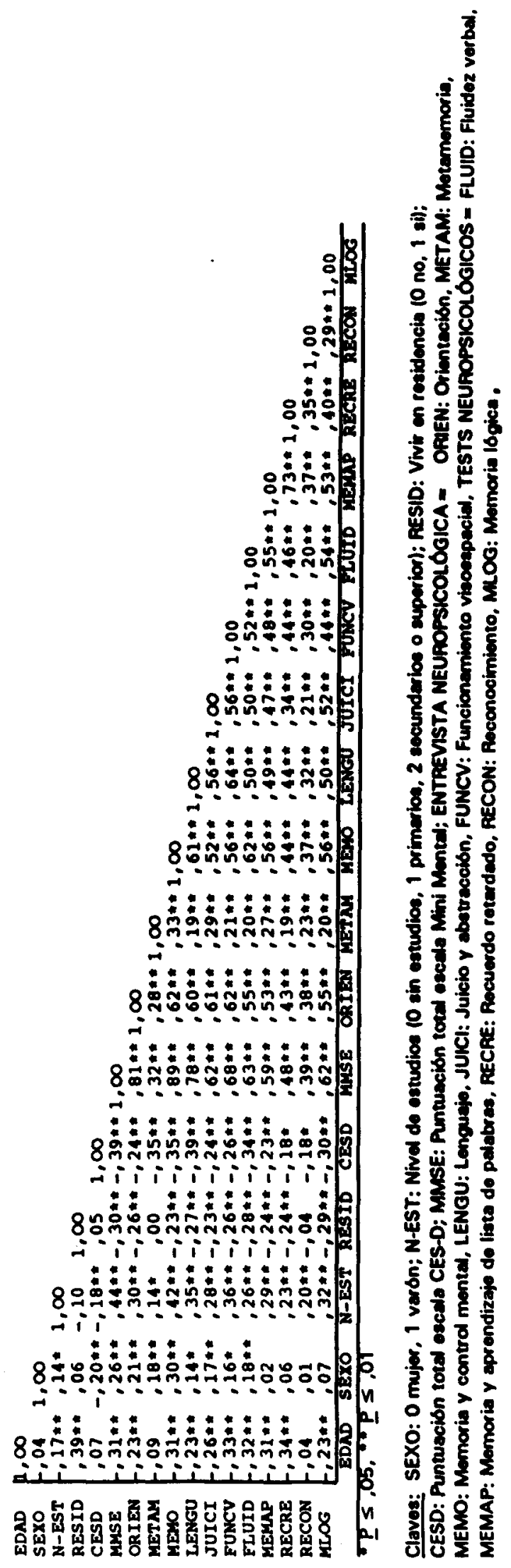


variables cognitivas están muy relacionas entre sí y también con la puntuación del MMSE, la edad, el nivel de estudios y, en menor medida, el género.

\section{DISCUSIón}

Los resultados de este estudio permiten establecer diversas relaciones entre la depresión y las variables lugar de residencia, edad, género y nivel de estudios. Además nos confirman la idea de que determinadas tareas cognitivas, condicionadas por la edad, el género $y$, fundamentalmente, por el nivel de estudios, probablemente puedan ser predictoras de la depresión.

En primer lugar, los resultados sobre la depresión, conseguidos mediante el CES-D, muestran una enorme semejanza con los obtenidos con los criterios del DSM-III-R, que también hemos utilizado. Esta coincidencia de resultados avala la utilidad de la escala CESD para la evaluación de la sintomatología depresiva en la población de ancianos, tal y como ya se habia puesto de relieve en diversos trabajos (Gatz y Hurwicz, 1990, Hertzog et al. 1990). Pero la escala CES-D no sólo ha demostrado su utilidad en la evaluación clínica de la depresión en la vejez, sino que nos ha permitido comparar nuestros resultados con los obtenidos en muestras culturalmente muy diferentes. Asi vemos que la media de la puntuación total del CES(D en nuestra muestra (15.03) no difiere significativamente de las obtenidas con ancianos americanos, tanto blancos (15.32) como negros (16.35), y taiwaneses (14.72), y es ligeramente superior a la de los japoneses (13.20) (Krause y Liang, 1992). Si distribuimos a los sujetos de nuestra muestra en los mismos inter-valos de edad utilizados por Gatz y Hurwicz (1990), se comprueba que, aunque la media total del CES(D para el grupo de edad entre 55 y 69 años es mayor en nuestro trabajo (14.52) que en la muestra americana (7.66), para el grupo de 70 años o más hay gran coincidencia entre nuestros datos (15.19) y los del citado trabajo (14.43).

Por otra parte, la diferencia de prevalencia de la depresión entre los que viven en la residencia y los que viven en su domicilio no es esta-dísticamente significativa, aunque las ta- sas son mayores en los primeros. Las mujeres presentan en ambos ambientes una prevalencia mayor. Pero se dan diferencias mayores entre los hombres de los dos ambientes, que entre las mujeres. La mayor tasa presente de depresión en los residentes, pero sin que las diferencias sean significativas, es similar a la encontrada por otros autores, como Lazaro (1991). Otros estudios, sin embargo, como el de Rojas, Heras, Reig, y Angeles (1991) con una muestra de ancianos de una residencia geriátrica, donde un alto porcentaje manifestaban sentirse solos, abandonados por su familiares y poco visitados, muestran una tasa de depresión del $63 \%$; un porcentaje muy alto en comparación con el $40,6 \%$ de nuestros datos. Las diferencias encontradas en distintos estudios probablemente se justifiquen por los diversos tipos de "institucionalización" estudiados en cada caso. En consecuencia, pensamos que se pueden encontrar grandes dificultades en la comparación de sujetos que viven en diferentes instituciones, si no se definen antes claramente las características de la "institución" residencial, que puedan ser relevantes para tal comparación.

Respecto a la edad, encontramos diferencias significativas entre los tres intervalos de edad en la puntuación total del CES-D, además de una representación gráfica en forma de U. Las altas puntuaciones en el grupo de menores de $7 \mathrm{C}$ años probablemente estén relacionadas con el inicio de la vejez sociologica y con la necesidad por parte de los sujetos de asumir nuevos roles. Por otro parte, el incremento, aun mayor, de las puntuaciones en los mayores de 80 años suponemos que está asociado a que es una edad crítica en el proceso de envejecimiento para el deterioro general $y$, en concreto, para el cognitivo, y a la acumulación con los años de experiencias negativas, especialmente de soledad y de pérdida.

En cuanto el género, la prevalencia de la sintomatologia depresiva es significativamente mayor, en todos los intervalos de edad, dándose una presencia 1.6 veces mayor en las mujeres que en los hombres, lo cual confirma la cifra de 1.5 veces mayor en las mujeres encontrada en otros trabajos (Weissman y Klerman, 1985; Bebbington, 1988; Anthony y Aboraya, 1992). Existe una controversia, todavía no resuelta, con respecto a las diferencias 
de salud mental en razón del género. Nuestros resultados avalan los obtenidos por otros autores (Gatz y Hurwicz 1990; Gatz, 1994). No obstante, pensamos que estas diferencias no se derivan directamente del género, sino de ciertas variables asociadas al género, como el mayor deterioro cognitivo de la mujer o su nivel de estudios más bajo en comparación con el hombre. La correlación entre la sintomatologia depresiva y el deterioro cognitivo, mayor en las mujeres que en los hombres, podemos corroborarlo por el hecho de que una de las variables que mejor explican y predicen la depresión en las mujeres es el deterioro cognitivo. A su vez, la variable que mejor predice el deterioro cognitivo es el nivel de estudios, que en nuestra muestra es inferior en la mujer que en el hombre. De hecho, cuando se iguala el nivel de estudios en hombres y mujeres, desaparecen también las diferencias por género en el deterioro cognitivo (Montanés, Latorre y Castejón, 1996).

La interrelación anteriormente indicada entre depresión y deterioro cognitivo es especialmente importante a partir de los 80 años. Si separamos depresión y deterioro cognitivo en el diagnóstico los datos cambian de la siguiente forma: La prevalencia de la depresión sin deterioro cognitivo disminuye con la edad, desapareciendo la curva en U; la prevalencia del deterioro cognitivo sin depresión aumenta; y la prevalencia de sujetos que muestran al mismo tiempo depresión y deterioro cognitivo también aumenta. Este último aumento del diagnóstico común (sujetos que muestran al mismo tiempo depresión y deterioro cognitivo) incrementa la probabilidad de confusión, cuanto mayor sea el sujeto, especialmente a partir de los 80 años, acerca de cuál es el problema principal, la depresión o el deterioro. La bibliografia sobre el tema confirma esta dificultad. En estudios comunitarios realizados con el MMSE, se ha podido comprobar que de los sujetos que puntúan 23 o menos, sólo la tercera parte es finalmente diagnosticada de demencia, el resto o no presenta ninguna alteración o se incluye en otros problemas psiquiátricos, fundamentalmente depresión (Folstein et al., 1975; Morris y Kopelman, 1992).

Esto nos hace pensar que conforme el anciano tiene mayor edad, la evaluación de la depresión debe acompañarse de pruebas neuropsicológicas relacionadas con el deterio- ro cognitivo, ya que el clásico problema del diagnóstico diferencial en los ancianos estriba en distinguir demencia de depresión y demencia del delirio, y estos tres de los estados normales de la edad. En este sentido, como señala Montorio (1994), los pre-requisitos de la evaluación de la depresión en personas mayores pasan por la evaluación de una demencia potencial y de la existencia de otros componentes fisicos o farmacológicos que pueden estar asociados al cuadro depresivo.

Entre las diversas explicaciones que se pueden dar al problema de la interrelación entre depresión y deterioro cognitivo están las siguientes: primera, los estados emocionales, como la ansiedad y la depresión, tienen un impacto especial sobre el rendimiento cognitivo en la vejez a diferencia de otras edades (Deptula et al., 1993); segunda, las personas mayores con deterioros cognitivos tienen a menudo una depresión concomitante (Reifler et al., 1982); tercera, en algunos estudios prospectivos pequeños se ha encontrado que algunos pacientes que se quejan de su memoria, pero que son diagnosticados como deprimidos, evolucionan realmente a casos de demencia (Reding. Haycox, y Blass, 1985); y cuarta, la variable educación está implicada en la ejecución de las pruebas neuropsicológicas, en el deterioro cognitivo y en la depresión. La especial confluencia de estas circunstancias, en la vejez, impide una respuesta univoca a los interrogantes que se suscitan. Los datos, obtenidos con nuestro trabajo, permiten constatar la importancia de determinadas tareas cognitivas para discriminar mejor entre depresión y deterioro cognitivo, y la relación que el nivel de estudios tiene sobre esas tareas.

A la vista de nuestros resultados, sobre el poder discriminativo de las distintas tareas cognitivas entre vejez normal, depresión y deterioro, podemos concluir que: 1) las pruebas de naturaleza verbal, como la fluidez verbal y la memoria lógica, discriminan mejor el deterioro cognitivo, tanto en sujetos con depresión como sin ella; 2) cuando se comparan los ancianos sin depresión con los que sólo presentan deterioro cognitivo, aparecen diferencias significativas en todas las pruebas, especialmente en fluidez verbal; 3) cuando se comparan los ancianos con depresión y con deterioro cognitivo, el recuerdo demorado de una 
lista de palabras se convierte en la prueba que mejor discrimina; y 4) cuando se compara a los sujetos normales con los deprimidos, las diferencias sólo existen en la tarea de metamemoria.

Estos resultados requieren algunos comentarios. En primer lugar, el hecho de que las tareas de naturaleza verbal, como la fluidez y la memoria lógica discriminen mejor el deterioro cognitivo de lo que no lo es concuerda con los resultados obtenidos por diversos autores (Cummings, Benson y Hill, 1985; Kertesz, Appel y Fisman, 1986; Hart, Kwentus, Taylor y Harkins, 1987; Emery, 1992). En segundo lugar, el hecho de que la metamemoria se deteriore significa-tivamente en los deprimidos muestra por qué muchos sujetos con depresión acuden a las consultas generales o especializadas con el temor a sufrir algún tipo de demencia, resultado que confirma lo hallado por otros autores (Bazargan y Barbre, 1994; McDougall, 1994; Dellefield y McDougall, 1996). En tercer lugar, los resultados mostrados en la matriz de correlaciones, acerca de la relación entre la metamemoria y el rendimiento, van en la línea de los trabajos que sugieren la existencia de una relación entre la metamemoria y la ejecución de tareas cognitivas (Zelinski et al., 1980; Dellefield y McDougall, 1996; Guirado y Latorre, 1996). En cuarto lugar, cabe destacar la prueba de recuerdo demorado como evaluación complementaria al MMSE para diferenciar entre casos "puros" de demencia y depresión. En este sentido apuntan diversos trabajos senalando que, mientras los sujetos deprimidos muestran un "almacenamiento" normal cuando el aprendizaje inicial se iguala a los de control, en los sujetos con demencia tipo Alzheimer se da un olvido muy rápido, atribuido a las lesiones en el hipocampo, que en esta demencia ocurren ya en las primeras etapas (para una discusión sobre el tema ver Morris y Kopelman, 1992). Estos resultados, aunque no definitivos, pueden ser indicativos de qué pruebas son mejores a la hora de diferenciar los diversos estados estudiados en la vejez.

Nuestro trabajo confirma la estrecha relación existente entre el nivel de estudios, el deterioro cognitivo y la depresión, ya que en los análisis realizados la depresión y el deterioro cognitivo aumentan en los niveles educativos más bajos y viceversa. Además, el poder discriminativo de las pruebas cognitivas utiliza- das varia apreciablemente según el nivel de estudios de los sujetos: La memoria lógica, por ejemplo, es la prueba que mejor discrimina entre deprimidos y no deprimidos en sujetos sin estudios; mientras discrimina mal entre sujetos con estudios. Por el contrario, la fluidez verbal discrimina mejor la depresión en los sujetos con estudios, que en los que carecen de ellos.

Por nuestra parte creemos que el nivel de estudios más que relacionarse directamente con la depresión, lo hace indirectamente a través de variables intermedias como el estatus socioeconómico y las habilidades de afrontamiento desarrolladas a lo largo del ciclo vital. Estos resultados deberian completarse con estudios que utilicen la metodologla longitudinal y secuencial, para evitar las posibles confusiones entre los efectos de la edad y los de la cohorte. Otro posibilidad sería realizar análisis de variables latentes, ampliando de forma importante la muestra de sujetos con estudios medios o superiores, de los cuales adolece nuestra muestra.

Por último, creemos conveniente, a la luz de nuestros resultados, alertar sobre la necesidad de extremar las precauciones en la evaluación del deterioro cognitivo vs. depresión en los sujetos mayores de 75 años, con un bajo nivel de estudios. Como hemos podido ver, en el sector de la muestra sin estudios se reduce considerablemente el poder discriminativo de las pruebas debido al deterioro de las que tienen una importante carga verbal (v.g. fluidez verbal o memoria lógica). En el caso de trabajar con estos sujetos sería conveniente utilizar pruebas de recuerdo demorado, como las que figuran en la Escala de Memoria de Weschler (WMS-R, Weschler, 1987) que han demostrado su eficacia en estas circunstancias (Guirado y Latorre, 1996), para complementar las que nosotros hemos utilizado aqul.

\section{AGRADECIMIENTOS}

Este trabajo ha sido realizado con la financiación del INSERSO ( $\mathrm{M}^{\circ}$ de Asuntos Sociales) y de la Universidad de Castilla-La Mancha (1994-95). Asímismo agradecemos a la Dra. Margaret Gatz (Departament of Psychology, University of South California, Los Angeles, USA) que nos haya facilitado algunas de las pruebas utilizadas en la realización de este trabajo. 


\section{REFERENCIAS BIBLIOGRÁFICAS}

American Psychiatric Association (1987). Diagnostic and statistical manual of mental disorders ( $3^{*}$ Ed., rev.). Washigton, DC: Author.

American Psychiatric Association (1994). Diagnostic and statistical manual of mental disorders ( $4^{\mathrm{a}}$ Ed.). Washigton, DC: Author.

Andresen, E.M., Malmgren, J.A., Carter, W.B. y Patrick, D.L. (1994). Screening for depression in well older adults: Evaluation of a short form of the CES-D. American Journal Medicine, 10 (2), 77-84.

Anthony, J.C. y Aboralla, A. (1992). The epidemiology of selected mental disorders in later life. En J.Birren, R.B. Sloane y G.D.Cohen (Eds.), Handbook of mental health and aging ( $2^{a}$ ed., pp. 28-73). San Diego: Academic Press.

Bazargan, M. y Barbre, A. (1994). The effects of depression, health status, and stressful lifeevents on self-reported memory problems among aged blacks. International Joumal Aging and Human Development, 38 (4), 351-362.

Beato, M.S. y Fernández, A. (1995). Memoria explicita $\theta$ implicita en pacientes depresivos: diferencias entre pruebas perceptivas y conceptuales. Cognitiva, 7(1), 51-66.

Bebbington, P.E. (1988). The social epidemiology of clinical depression. En A.S. Henderson y G.D. Burrows (Eds.), Handbook of social psychiatry (pp. 87-102). Amsterdam: Elsevier Science Publishers.

Berkman, L.F., Berkman, C.S., Kasl, S., Freeman, H.D. y Leo, L. (1986). Depressive symptoms in relation to physical health and function in the elderly. American Joumal of Epidemiology, 124, 372-388.

Bermejo, F. y Colmenarejo, C. (1993). Prevalencia de demencia y deterioro cognitivo. En F. Bermejo (Coord.), Nivel de salud y deterioro cognitivo en los ancianos, (pp. 99-118). Barcelona: S.G. Editores y Fundación Caja Madrid.

Bermejo, F. y García de Blas, F. (1993). Relación entre nivel cognitivo y salud. En F. Bermejo (Coord.). Nivel de salud y deterioro cognitivo en los ancianos (pp. 119-128). Barcelona, S.G. Editores y Fundación Caja Madrid.

Berry, J.M., Storandt, M. y Coyne. A. (1984). Age and sex differences in somatic complaints associated with depression. Journal of Gerontology, 39, 465-467.

Blazer, D., Hughes, D.C. y George, L.K. (1987). The epidemiology of depression in an elderly community population. The Gerontologist, 27 . 281-287.

Boyd, J.H., Weissman, M.M., Thompson, W.D. y Myers, J.K. (1982). Screening for depression in a community sample: understanding the discrepancies between depression symptom and diagnostic scales. Archives of General Psyquiatry, 39, 1195-1200.

Brown, D.R., Milbum, N.G. y Gary, L.E. (1992). Symptoms of depression among older African Americans: an analysis of gender differences. Gerontologist, 32, 789-795.

Buendia, J. y Riquelme, A. (1995). Trastornos asociados a la vejez. En A. Belloch, B. Sandín y F. Ramos (Dirs.), Manual de Psicopatologla, (Vol. 2, pp. 747-767). Madrid: McGraw-Hill.

Busse, E.W. (1992). Quality of life: affect and mood in late life. En M. Bergener, K. Hasegawa, S.I. Finkel y T. Nishimura (Eds.), Aging and mental disorders. International perspectives, (pp. 3855). Nueva York: Springer.

Cohen, G. D. (1990). Psychopathology and mental health in the mature and elderly adult. En J.E. Birren y K. W. Schaie (Eds.), Handbook of the psychology of aging (3* ed., pp. 359-371). San Diego: Academic Press.

Colsher, P.L. y Wallace, R.B. (1991). Epidemiologic considerations in studies of cognitive function in the elderly: methodology and nondementing acquired dysfunction. Epidemiological Review, 13, 1-27.

Comstock, G.W. y Helsing, K.J. (1976). Symptoms of depression in two communities. Psychological Medicine, 6, 551-563.

Costa, P.T., McCrae, R.R. y Locke, B.Z. (1990). Personality factors. En J.C. Coroni-Huntley, R.R. Huntdley, J.J. Feldman (Eds.), Heatth status and well-being of the eldenly, (pp. 210-220). New York: Oxford University Press.

Cummings, J.L., Benson, D.F. y Hill, M.A. (1985). Aphasia in dementia of the Alzheimer type. Neurology, 35, 394-397.

Davis, H. (1979). The self-schema and subjective organization of personal information in depression. Cognitive Therapy and Research, 3, 415-425.

Dawson, M., Schell, A. y Catania, J. (1977). Automatic correlates of depression and clinical improvement following electroconvulsive shock therapy. Psychophysiology, 14, 564-578.

Dellefield, K.S. y McDougall, G.J. (1996). Increasing metamemory in older adults. Nursing Research, 45 (5), 284-290.

Deptula, D., Singh, R. y Pomara, N. (1993). Aging, emotional states, and memory. American Joumal of Psychiatry, 150, 429-434.

Eaton, W.W. y Kessler, L.G. (1981). Rates of symptoms of depression in a national sample. American Journal of Epidemiology, 144, 528-538. 
Ellis, H.C. y Ashbrook, P.W. (1988). Resource allocation model of the effects of depressed mood states on memory. En K. Fiedler y J. Forgas (Eds.), Affect, cognition and social behavior, (pp. 25-43). Toronto: Hogrefe.

Emery, O.B. (1992). Interaction of language and memory in major depression and senile dementia of Alzheimer's type. En L. Băckman (Ed.). Memory functioning in dementia, (pp. 175-199). Amsterdam: North-Holland.

Fernández-Ballesteros,R., |zal, M., Montorio, I., González, J.L. y Diaz, P. (1992). Evaluación e intervención psicológica en la vejez. Madrid: Martínez Roca.

Fingerman, K.L. y Perlmutter, M. (1994). Self-Ratings of past, present, and future cognitive performance across adulthood. Intemational Joumal Aging and Human Development, 38 (4), 363-382

Folstein, M.F., Folstein, S.E. y McHugh, P.R. (1975). Mini Mental State: a potential method for grading the cognitive state of the patients for the clinician. Joumal of Psychiatric Research, 12, 189-198.

Frerichs, R.R., Aneshensel, C.S. y Clark, V.A. (1981). Prevalence of depression in Los Angeles County. American Joumal of Epidemiology, 113, 691. 699.

Garcia, M. y Marks, G. (1989). Depressive symptomatology among mexican-american adults: an examination with the CES-D scale. Psychiatry Research, 27, 137-148.

Gatz, M. (1994). Dificultades en la salud mental de los adultos mayores. Conferencia impartida en el Campus Universitario de Albacete. Inédita.

Gatz, M. y Hurwicz, M.L. (1990). Are old people more depressed? Cross-sectional data on Center for Epidemiological Studies Depression Scale factors. Psychology and Aging, 5 (2), 284-290.

Gatz, M., Johansson, B., Pedersen, N., Berg, S., y Reynolds, Ch. (1993). A cross-national self-report measure of depressive symptomatology. Intemational Psychogeriatrics, 5(2), 147-156.

González, H. y Ramos, F. (1995). Las demencias seniles. En A. Belloch, B. Sandin y F. Ramos (Dirs.), Manual de Psicopatologia, (Vol. 2 pp. 769-790). Madrid: McGraw-Hill.

Guirado, P. y Latorre, J.M. (1996). Rendimiento en pruebas de memoria de ancianos normales y con demencia. En M. Marin (Coord.), Sociedad y Educación, (pp. 641-654). Sevilla: Eudema.

Hankin, J.R. y Locke, B.Z. (1982) The persistence of depressive symptomatology among prepaid group practice enrollees: an exploratory study. American Joumal of Public Health, 72, 10001007.

Hart, R.P., Kwentus, J.A., Taylor, J.R. y Harkins, S.W. (1987). Rate of forgetting in dementia and depression. Joumal of Consulting and Clinical Psychiatry, 55, 101-115.
Hertzog, C. (1989). Using confirmatory factor analysis for scale development and validation. En M. Powell y A.R. Hertzog (Eds.), Special research methods for gerontology, (pp. 281-306). Nueva York: Baywood.

Hertzog, C., Van Alstine, J., Usala, P.D., Hultsch, D.F. y Dixon, R. (1990). Measurement properties of the Center for Epidemiological Studies Depression Scale (CES-D) in older populations. Psychological Assessment, 2, 64-72.

Himmelfarb, S. y Murrel, S.A. (1983). Reliability and validity of five mental health scales in older persons. Journal of Gerontology, 38, 333-339.

Huyck, M.H. (1990). Gender differences in aging. En J.E. Birren y K. W. Schaie (Eds.), Handbook of the psychology of aging (3* ed., pp. 124-132). San Diego: Academic Press.

Kalish, R.A. (1991). La vejez. Perspectivas sobre el desarrollo humano. Madrid: Pirámide (Publicación original, 1982).

Kennedy, G.J., Kelman, H.R., Thomas, C., Wisniewski, W., Metz, H. y Bijur, P.E. (1989). Hierarchy of characteristics associated with depressive symptoms in an urban elderly sample. American Journal of Psychiatry, 146, 220 . 225.

Kertesz, A., Appel, J. y Fisman, M. (1986). The dissolution of language in Alzheimer's disease. Canadian Journal of Neurological Sciences, 13, 415-418.

Krause, N. (1986). Stress and sex differences in depresive symptoms among older adults. Journal of Gerontology, 41, 727-731.

Krause, N. y Liang, J. (1992). Cross-cultural variations in depressive symptoms in later life. Intemational Psychogeriatrics, 4(2), 185-202.

Lazaro, M.L. (1991). Areas afectiva y cognitiva en la vejez. Predictores de mortalidad. Comunicación Psiquiátrica, 16, 95-116.

Lebowitz, B.D. y Niederehe, G. (1992). Concepts and issues in mental health and aging. En J.Birren, R.B. Sloane y G.D.Cohen (Eds.), Handbook of mental health and aging ( $2^{\bullet}$ ed., pp. 3-27). San Diego: Academic Press.

Liptzin, B. (1992) Nursing home care. En J.Birren, R.B. Sloane y G.D.Cohen (Eds.), Handbook of mental health and aging (2* ed. pp. 833-853). San Diego: Academic Press.

Massan, P.J., Butters, N.M. y Delis, D.C. (1994). Some comparisons of the verbal leaming deficit in Alzheimer demetia, Huntington disease, and depression. En V.O.B. Emery y T.H. Oxman (Eds.), Dementia, (pp. 232-248). Baltimore: Johns Hopkins Univ. Press.

McDougall, G.J. (1994) Predictors of metamemory in older adults. Nursing Research, 43 (4), 212218. 
Montañés, J., Latorre, J.M. y Castejón, J.L. (1996). Evaluación del deterioro cognitivo en la vejez. En M. Marin (Coord.) Sociedad y Educación, (pp. 667-676). Sevilla: Eudema.

Montorio, I. (1994). La persona mayor. Guía aplicada de evaluación psicológica. Madrid: INSERSO, Ministerio de Asuntos Sociales.

Morris, J.C., Mohs, R.C., Rogers, H., Fillenbaum, G. y Heyman, A. (1988). Consortium to Estabish a Registry for Alzheimer's Disease (CERAD) clinical and neuropsychological assessment of Alzheimer's disease. Psychopharmacology Bulletin, 24, 641-652.

Morris, R.G. y Kopelman, M.D. (1992). The neurospychological assessment of dementia. En J.R. Crawford, D.M. Parker y W.W. McKinlay (Eds.). A handbook of neuropsychological assessment, (pp. 295-314). Hillsdale: LEA.

Mortimer, J.A. (1993) What are the risk factors for dementia?. En F. Huppert, C. Brayne. y D. O'Connor (Eds.), Dementia and normal aging, (pp. 78-97). Cambridge: Cambridge University Press.

Murrel, S.A., Himmelfarb, S. y Wright, K. (1983). Prevalence of depression and its correlates in older adults. American Journal of Epidemilogy, 177, 173-185.

Myers, J.K. y Weissman, M.M. (1980) Use of a selfreport symptom scale to detect depression in a community sample. American Journal of Psychiatry, 137, 1081-1084.

Newmann, J.P. (1989). Aging and depression. Psychology and Aging, 4, 150-165.

O'Connor, D.W., Pollit, P.A. y Roth, M. (1990). Memory complaints and impairment in normal, depresed, and dement elderly persons identified in a community survey. Archives of General Psyquiatry, 47, 224-227.

Pedersen, N.L., Gatz, M., Winblad, B., Pearson, C.G, y Berg, S. (1988). Dementias in Swedish twins. Comunicación presentada a la Primera Conferencia Internacional sobre la Enfermedad de Alzheimer y desórdenes relacionados, Las Vegas, NV. Resúmen publicado en Alzheimer Disease and Associated Disorders, 2, 283.

Radloff, L.S. (1977). A self-report depression scale for research in the general population. Applied Psychological Measurement, 1, 385-401.

Radloff, L.S. y Teri, L. (1986). Use of the Center of Epidemiological Studies Depresion Scale with older adults. Clinical Gerontologist, 5, 119-136.

Reding. M., Haycox, J. y Blass, J.(1985). Depression in patients referred to a dementia clinic, $A$ three- year prospective study. Archives of Neurology, 42, 894-896.

Reifler, B.V., Larson, E. y Hanley, R. (1982). Coexistence of cognitive impairment and depression in geriatric outpatients. American Joumal of Psychiatry, 139, 623-626.

Reisberg, B., Ferris, S.M., Steinberg, G., Shulman, E., de León, M.J. y Sinaiko, E. (1989). Longitudinal study of dementia patients and aged controls: and approach to methodological issues. En M.P.Lawton y A.R.Herzg (Eds), Special research methods for gerontology. (pp. 195-232). Amityville, N.Y.: Baywood Publishing Company, Inc.

Roberts, R.E. y Vernon, S.W. (1983). The center for epidemiological studies scale. Its use in a community sample. American Journal of Psychiatry, 140, 41-46.

Rodríguez-Naranjo, C. y Godoy, A. (1997). Factores que predicen la eficacia de diferentes tratamientos para la depresión leve o disforia. Psicotema, 9(1), 105-117.

Rojas, E., Heras, F.J., Reig, M.J. y Angeles, F. (1991). Depresión y factores sociofamiliares en ancianos de una residencia geriátrica. Psicopatologia, 11(1), 20-24.

Ruiz, M., Martin, F., Serrano, V. y Morales, M. (1988). Depresiones en las diferentes edades de la vida. Psiquis, 9(2), 11-22.

Steen, B. (1992). Psychosocial and cultural aspects of dementia: a european perspective. En M. Bergener, K. Hasegawa, S.I. Finkel y T. Nishimura (Eds.), Aging and mental disorders. Intemational perspectives, (pp. 124-133). Nueva York: Springer.

Sweeney, J.A., Stokes, P., Wetzler, S., y Kocsis,J. (1989). Cognitive functioning in depression. Joumal of Clinical Psychology, 45(6), 836-842.

Vázquez, C. y Sanz, J. (1995). Trastomos del estado de ánimo. aspectos clínicos. En A. Belloch, B. Sandín y F. Ramos (Dirs.), Manual de Psicopatología, (Vol. 2 pp. 769-790). Madrid: McGraw-Hill.

Watkins, P.C., Mathews, A., Williamson, D.A., y Fuller, R.D. (1992). Mood congruent memory in depression: Emotional priming or elaboration?. Journal of Abnormal Psychology, 101, 581-586.

Weschler, D. (1987). Weschler Memory ScaleRevised. Nueva York: Psychological Corporation.

Weissman, M.M. y Klerman, G,L, (1985). Gender and depression. Trends in Neuroscience, 8, 416420.

Zelinski, E.M., Gilewski, M.J. y Thompson, L.W. (1980). Do laboratory tests relate to selfassessment of memory ability in the young 
and old?. En L.W.Poon, J.L.Fozard, L.S. Cermak, D.Arenberg y L.W. Thompson (Eds.). New directions in memory and aging (pp. 519-544). Hillsdale, N.J.: Eribaum.
Zhang, M., Katzman, R. y Salmon, D. y otros (1990). The prevalence of dementia and Alzheimer's disease in Shanghai, China: Impact of age, gender, and education. Annual Review of Neurology, 27, 428-437. 